\title{
Article
}

Mycosphere

\section{Saprobic Botryosphaeriaceae, including Dothiorella italica sp. nov., associated with urban and forest trees in Italy}

\author{
Dissanayake AJ ${ }^{1,2}$, Camporesi E ${ }^{3}$, Hyde KD ${ }^{2}$, Yan JY' ${ }^{1}$ Li XH ${ }^{1}$ \\ ${ }^{1}$ Beijing Key Laboratory of Environmental Friendly Management on Fruit diseases and Pests in North China, Institute \\ of Plant and Environment Protection, Beijing Academy of Agriculture and Forestry Sciences, Beijing 100097, People's \\ Republic of China \\ ${ }^{2}$ Center of Excellence in Fungal Research, School of Science, Mae Fah Luang University, Chiang Rai 57100, Thailand \\ ${ }^{3}$ A.M.B. Gruppo Micologico Forlivese "Antonio Cicognani”, Via Roma 18, Forli, Italy
}

Dissanayake AJ, Camporesi E, Hyde KD, Yan JY, Li XH 2017 - Saprobic Botryosphaeriaceae, including Dothiorella italica sp. nov., associated with urban and forest trees in Italy. Mycosphere 8(2), 1157-1176, Doi 10.5943/mycosphere/8/2/7

\begin{abstract}
A collection of saprobic botryosphaeriaceous isolates (from dead aerial branches, stems, and dead leaves) was obtain from urban and forest tree species in Italy. A total of 52 isolates were characterized by multi-locus sequence analysis of the internal transcribed spacer region (ITS) and partial sequences of the translation elongation factor 1-alpha (TEF). According to morphological characteristics and analysis of multi-gene sequence data, 14 species in Botryosphaeriaceae belonging to five genera, viz. Botryosphaeria, Diplodia, Dothiorella, Eutiarosporella and Neofusicoccum were identified, including Dothiorella italica sp. nov. The other known species isolated were Botryosphaeria dothidea, B. auasmontanum, Diplodia alatafructa, Di. crataegicola, Di. mutila, Di. sapinea, Di. seriata, Dothiorella omnivora, Do. rhamni, Do. sarmentorum, Do. sempervirentis, Eutiarosporella dactylidis and Neofusicoccum parvum. Of these, Botryosphaeria dothidea and Diplodia seriata were the most prevalent species among the isolates. Several new host-fungus relationships were established. This study indicates the fungal family Botryosphaeriaceae seems to be common and widespread on a broad range of hosts in Italy.
\end{abstract}

Key words - Botryosphaeriales - Botryosphaeria - Diplodia - Eutiarosporella - multigene phylogeny - Neofusicoccum

\section{Introduction}

The family Botryosphaeriaceae Theiss. \& Syd. comprise 23 genera found on a wide range of plant hosts (Dissanayake et al. 2016b). Many botryosphaeriaceous taxa can exist as endophytes (Slippers \& Wingfield 2007), or are primary or opportunistic pathogens or saprobes (Schoch et al. 2006, Mehl et al. 2014) and are widespread in tropical and temperate regions (Phillips et al. 2013, Dissanayake et al. 2016b). Almost all Botryosphaeriaceae species are plant-associated and most of them cause disease symptoms on ecologically and economically significant plants in forestry and agriculture (Hyde et al. 2013, 2014, Phillips et al. 2013, Slippers et al. 2013). Botryosphaeriaceae consists of many well-known plant pathogens such as Botryosphaeria dothidea (Moug.) Ces. \& De Not., Diplodia seriata De Not., Dothiorella sarmentorum (Fr.) A.J.L. Phillips, A. Alves \& J. Luque, Lasiodiplodia theobromae (Pat.) Griffon \& Maubl. Neofusicoccum parvum (Pennycook \& 
Samuels) Crous, Slippers \& A.J.L. Phillips and many others causing severe infections (Slippers et al. 2013, Yan et al. 2013, Hyde et al. 2014). Botryosphaeria Ces. \& De Not. species are frequently associated with diseases such as dieback, cankers, shoot blights, leaf spots, fruit and seed rots. Diplodia Fr. species are associated with diverse symptoms such as cankers, dieback, gummosis, fruit rots and twig blight (Lazzizera et al. 2008, Giambra et al. 2016, Linaldeddu et al. 2016c). Though several Diplodia species such as Di. mutila (Fr.) Mont., Di. pseudoseriata C.A. Pérez, Blanchette, Slippers \& M.J. Wingf., Di. scrobiculata J. de Wet, Slippers \& M.J. Wingf. and Di. seriata have an extensive host range, other Diplodia species have been described from very limited plant species and may be host-specific (Phillips et al. 2013, Dissanayake et al. 2016b). The genus Dothiorella Sacc. is widely spread in woody hosts world-wide (Dissanayake et al. 2016a) and morphologically similar to Spencermartinsia A.J.L. Phillips, A. Alves \& Crous in asexual morph, but treated as a distinct genus based on the presence of apiculi on ascospores of Spencermartinsia (Phillips et al. 2008, 2013). However, this separation was recently perceived as uncertain (Slippers et al. 2014). Except Do. iberica A.J.L. Phillips, J. Luque \& A. Alves, Do. omnivora B.T. Linaldeddu, A. Deidda \& B. Scanu and Do. sarmentorum; all other Dothiorella species were reported from very few plant species (Phillips et al. 2013, Dissanayake et al. 2016b). Compared to other genera of Botryosphaeriaceae, species of Eutiarosporella Crous have different culture characteristics and conidial morphology (Jami et al. 2012, Thambugala et al. 2014, Crous et al. 2015). Although recent studies have reported Eutiarosporella species from woody hosts (Jami et al. 2012, 2014), traditionally they have been associated with family Poaceae (Sutton \& Marasas 1976, Nag Raj 1993, Li et al. 2016). The genus Neofusicoccum Crous, Slippers \& A.J.L. Phillips comprises species with widespread geographical and plant host distribution, some of them of economic importance (Lopes et al. 2017). Although Neofusicoccum species are typically endophytes, under stress environments certain species can cause deterioration and dieback symptoms (Crous et al. 2006, Slippers \& Wingfield 2007).

Before 2008, with exception of a few studies, all Botryosphaeriaceae species reported from Italy were identified based on morphological characters (Alves et al. 2004, Phillips et al. 2005, Linaldeddu et al. 2006, 2007), thus some of these reports are doubtful. Since 2008, numerous Botryosphaeriaceae species have been reported from Italy based on molecular data, either from symptomatic or asymptomatic native trees and/or from non-native trees (Lazzizera et al. 2008, Spagnolo et al. 2011, Ismail et al. 2013, Li et al. 2014, Linaldeddu et al. 2014, 2015, 2016a, b, c, Carlucci et al. 2015, Pavlic-Zupanc et al. 2015, Giambra et al. 2016). However, less is known regarding Botryosphaeriaceae species in the provinces of Forlì-Cesena, Arezzo and Perugia, in Italy. Thus, the aim of the present study was to explore the species composition, host range and distribution of saprobic Botryosphaeriaceae fungi in multiple plant species in Italy.

\section{Materials \& Methods}

\section{Sample collection, fungal isolation and morphological characterization}

Since 2012 to 2016, 52 Botryosphaeriaceae specimens were collected (Table 1) from dead aerial branches, stems and leaves of 34 host plants belonging to 23 host families (Adoxaceae, Asteraceae, Betulaceae, Celastraceae, Cornaceae, Cupressaceae, Fabaceae, Fagaceae, Magnoliaceae, Malvaceae, Meliaceae, Oleaceae, Pinaceae, Poaceae, Ranunculaceae, Rhamnaceae, Rosaceae, Rubiaceae, Salicaceae, Sapindaceae, Tamaricaceae, Ulmaceae and Vitaceae). These specimens were collected from plants in natural forest ecosystems and in urban environments in three provinces of Italy: Arezzo, Forlì-Cesena and Perugia. Specimens were observed and examined with a Motic SMZ 168 stereomicroscope. Fruiting bodies (ascomata and/or conidiomata) were detected in all collected samples and single spore isolations were made as previously described by Chomnunti et al. (2014). Conidial micro-morphological characteristics were observed with a Nikon ECLIPSE 80i compound microscope and images were captured with a Canon EOS 550D digital camera. 
Table 1 Botryosphaeriaceae species studied in this study (Fig. 1 to 5). Ex-type of the species introduced in this study is in bold.

\begin{tabular}{|c|c|c|c|c|c|c|c|c|}
\hline \multirow[t]{2}{*}{ Species } & \multirow[t]{2}{*}{$\begin{array}{c}\text { Strain } \\
(\text { MFLUCC) }\end{array}$} & \multirow[t]{2}{*}{ Host } & \multirow[t]{2}{*}{ Habit } & \multirow[t]{2}{*}{ Locality } & \multirow[t]{2}{*}{ Collector } & \multirow[t]{2}{*}{$\begin{array}{c}\text { Collection } \\
\text { date }\end{array}$} & \multicolumn{2}{|c|}{$\begin{array}{c}\text { GenBank accession } \\
\text { numbers }\end{array}$} \\
\hline & & & & & & & ITS & $\mathbf{E F}$ \\
\hline Botryosphaeria dothidea & $13-0292$ & Sambucus nigra & Dead aerial branch & Forlì-Cesena & N. Camporesi & 01.01 .2013 & MF398850 & MF398902 \\
\hline B. dothidea & $17-0940$ & Sambucus ebulus & Dead aerial stem & Forlì-Cesena & E. Camporesi & 15.03 .2013 & MF398851 & MF398903 \\
\hline B. dothidea & $17-0943$ & Galium sp. & Dead aerial stem & Forlì-Cesena & E. Camporesi & 10.02 .2014 & MF398852 & MF398904 \\
\hline B. dothidea & $17-0944$ & Carpinus betulus & Dead aerial branch & Forlì-Cesena & E. Camporesi & 19.02 .2014 & MF398853 & MF398905 \\
\hline B. dothidea & $15-0913$ & L. anagyroides & Dead aerial branch & Forlì-Cesena & E. Camporesi & 01.10 .2014 & MF398855 & MF398907 \\
\hline B. dothidea & $17-1069$ & Vitis vinifera & Dead aerial branch & Forlì-Cesena & E. Camporesi & 21.02 .2015 & MF398856 & MF398908 \\
\hline B. dothidea & $17-0960$ & Vitis vinifera & Dead aerial branch & Forlì-Cesena & E. Camporesi & 21.02 .2015 & MF398857 & MF398909 \\
\hline B. dothidea & $15-0927$ & P. menziesii & Dead land cone & Forlì-Cesena & E. Camporesi & 01.06 .2015 & MF398859 & MF398911 \\
\hline B. dothidea & $17-0973$ & Colutea arborescens & Dead aerial branch & Forlì-Cesena & E. Camporesi & 02.11 .2015 & MF398860 & MF398912 \\
\hline B. dothidea & $17-0986$ & Cornus sanguinea & Dead aerial branch & Forlì-Cesena & E. Camporesi & 24.11 .2015 & MF398861 & MF398913 \\
\hline B. dothidea & 17-0994 & Salix sp. & Dead aerial stem & Forlì-Cesena & E. Camporesi & 26.01 .2016 & MF398862 & MF398914 \\
\hline B. dothidea & $17-0945$ & Euonymus europaeus & Dead aerial branch & Forlì-Cesena & E. Camporesi & 20.02 .2014 & MF398854 & MF398906 \\
\hline B. auasmontanum & $15-0923$ & Rosa canina & Dead aerial branch & Forlì-Cesena & E. Camporesi & 07.04 .2015 & MF398858 & MF398910 \\
\hline B. auasmontanum & $17-1071$ & Alnus cordata & Dead aerial branch & Forlì-Cesena & E. Camporesi & 07.03 .2016 & MF398863 & MF398915 \\
\hline Diplodia alatafructa & $15-0924$ & Picea excels & Dead land cone & Forlì-Cesena & E. Camporesi & 12.04 .2015 & MF398880 & MF398932 \\
\hline Di. crataegicola & 13-0192 & Crataegus sp. & Dead aerial branch & Forlì-Cesena & E. Camporesi & 03.11 .2012 & MF398867 & MF398919 \\
\hline Di. crataegicola & $13-0289$ & Prunus sp. & Dead aerial branch & Forlì-Cesena & E. Camporesi & 23.12 .2012 & MF398870 & MF398922 \\
\hline Di. crataegicola & $15-0905$ & Tilia $s p$ & Dead aerial branch & Forlì-Cesena & E. Camporesi & 22.05 .2013 & MF398871 & MF398923 \\
\hline Di. mutila & $15-0917$ & Acer negundo & Dead aerial branch & Forlì-Cesena & E. Camporesi & 22.01 .2015 & MF398877 & MF398929 \\
\hline Di. mutila & $15-0918$ & Colutea arborescens & Dead aerial branch & Forlì-Cesena & E. Camporesi & 26.10 .2015 & MF398882 & MF398934 \\
\hline Di. sapinea & $17-0938$ & Picea excelsa & Dead land cone & Forlì-Cesena & E. Camporesi & 12.04 .2012 & MF398866 & MF398918 \\
\hline Di. sapinea & 13-0194 & Pinus pinaster & Dead aerial needles & Arezzo & E. Camporesi & 07.11 .2012 & MF398868 & MF398920 \\
\hline Di. seriata & $17-0936$ & Euonymus europaeus & Dead aerial branch & Forlì-Cesena & E. Camporesi & 08.04 .2012 & MF398865 & MF398917 \\
\hline Di. seriata & $15-0903$ & Quercus pubescens & Dead aerial branch & Forlì-Cesena & E. Camporesi & 02.12 .2012 & MF398869 & MF398921 \\
\hline
\end{tabular}




\begin{tabular}{|c|c|c|c|c|c|c|c|c|}
\hline Di. seriata & $17-0952$ & Rosa canina & Dead aerial spines & Forlì-Cesena & E. Camporesi & 06.10 .2014 & MF398872 & MF398924 \\
\hline Di. seriata & $17-0950$ & Rosa canina & Dead aerial stem & Forlì-Cesena & E. Camporesi & 17.10 .2014 & MF398876 & MF398928 \\
\hline Di. seriata & $17-0958$ & Sambucus nigra & Dead aerial branch & Forlì-Cesena & E. Camporesi & 07.02 .2015 & MF398878 & MF398930 \\
\hline Di. seriata & $15-0965$ & Rosa canina & Dead aerial spines & Forlì-Cesena & E. Camporesi & 25.03 .2015 & MF398879 & MF398931 \\
\hline Di. seriata & $17-0975$ & Magnolia grandiflora & Dead land leaf & Forlì-Cesena & E. Camporesi & 15.09 .2015 & MF398881 & MF398933 \\
\hline Di. seriata & $17-0989$ & Populus nigra & Dead aerial branch & Forlì-Cesena & E. Camporesi & 01.12 .2015 & MF398883 & MF398935 \\
\hline Di. seriata & $15-0760$ & Cornus sanguinea & Dead aerial branch & Forlì-Cesena & E. Camporesi & 02.03 .2012 & MF398864 & MF398916 \\
\hline Di. seriata & $15-0914$ & Rosa canina & Dead aerial spines & Arezzo & E. Camporesi & 13.10 .2014 & MF398875 & MF398927 \\
\hline Dothiorella omnivora & $17-0990$ & Cornus sanguinea & Dead aerial branch & Forlì-Cesena & E. Camporesi & 21.12 .2015 & MF398895 & MF398947 \\
\hline Do. rhamni & $17-1067$ & Tamarix gallica & Dead aerial branch & Forlì-Cesena & E. Camporesi & 01.12 .2012 & MF398888 & MF398940 \\
\hline Do. rhamni & $15-0922$ & Rhamnus alaternus & Dead aerial branch & Forlì-Cesena & E. Camporesi & 04.03 .2015 & MF398893 & MF398945 \\
\hline Do. sarmentorum & $15-0901$ & Clematis vitalba & Dead aerial branch & Forlì-Cesena & E. Camporesi & 26.03.2012 & MF398884 & MF398936 \\
\hline Do. sarmentorum & $15-0912$ & Ulmus minor & Dead aerial branch & Forlì-Cesena & E. Camporesi & 25.09 .2014 & MF398890 & MF398942 \\
\hline Do. sarmentorum & $15-0921$ & Vitis vinifera & Dead aerial branch & Forlì-Cesena & E. Camporesi & 21.02 .2015 & MF398892 & MF398944 \\
\hline Do. sarmentorum & $17-1000$ & Robinia pseudacacia & Dead aerial branch & Forlì-Cesena & E. Camporesi & 08.03 .2016 & MF398896 & MF398948 \\
\hline Do. sempervirentis & $17-1066$ & Cytisus sp. & Dead aerial branch & Perugia & E. Camporesi & 06.11 .2012 & MF398886 & MF398938 \\
\hline Do. italica & $17-1065$ & Cupressus sp. & Dead aerial branch & Forlì-Cesena & E. Camporesi & 13.04 .2012 & MF398885 & MF398937 \\
\hline Do. italica & $17-0954$ & Prunus sp. & Dead aerial branch & Perugia & E. Camporesi & 06.11 .2012 & MF398887 & MF398939 \\
\hline Do. italica & $15-0908$ & Rubus sp. & Dead aerial branch & Forlì-Cesena & E. Camporesi & 16.11.2013 & MF398889 & MF398941 \\
\hline Do. italica & 17-0951 & Rosa canina & Dead aerial branch & Forlì-Cesena & E. Camporesi & 17.10.2014 & MF398891 & MF398943 \\
\hline Do. italica & $17-0979$ & Melia azedarach & Dead aerial branch & Forlì-Cesena & E. Camporesi & 29.09 .2015 & MF398894 & MF398946 \\
\hline Do. italica & $17-1002$ & Ligustrum sp. & Dead aerial branch & Forlì-Cesena & E. Camporesi & 09.03 .2016 & MF398897 & MF398949 \\
\hline Eutiarosporella dactylidis & MFLU 15-3473 & A. elatius & Dead aerial stem & Forlì-Cesena & E. Camporesi & 03.11 .2013 & MF398898 & MF398950 \\
\hline Neofusicoccum parvum & $17-0961$ & Vitis vinifera & Dead aerial branch & Forlì-Cesena & E. Camporesi & 21.02 .2015 & MF398899 & MF398951 \\
\hline N. parvum & $17-0991$ & Salix sp. & Dead aerial branch & Forlì-Cesena & E. Camporesi & 18.12.2015 & MF398900 & MF398952 \\
\hline N. parvum & $17-1024$ & E. cannabinum & Dead aerial stem & Forlì-Cesena & E. Camporesi & 14.08 .2016 & MF398901 & MF398953 \\
\hline
\end{tabular}


Observations and photographs were made from materials mounted in water. Measurements were made with the Tarosoft $(\mathrm{R})$ Image Frame Work and images used for figures were processed with Adobe Photoshop CS3 Extended version 10.0. Cultures were incubated at $18^{\circ} \mathrm{C}$ in the dark and colony color was examined according to Rayner (1970) after $15 \mathrm{~d}$ of growth on PDA. Herbarium specimens are deposited in Mae Fah Luang University Herbarium (MFLU) while, extype living cultures are deposited at the Mae Fah Luang University Culture Collection (MFLUCC) in Thailand (Table 1).

\section{DNA extraction, amplification and phylogeny}

Mycelium from actively growing cultures was scraped from the surface of the plates. Total genomic DNA was extracted by the modified protocol of Dissanayake et al. (2016a). ITS and TEF gene regions were amplified using primer pairs ITS1/ITS4 (White et al. 1990) and EF-728F/EF986R (Carbone \& Kohn 1999) respectively.

PCR was performed in a BIORAD $1000^{\mathrm{TM}}$ Thermal Cycle in a total volume of $25 \mu \mathrm{l}$. The PCR mixtures contained TaKaRa Ex-Taq DNA polymerase $0.3 \mu 1,12.5 \mu 1$ of $2 \times$ PCR buffer with $2.5 \mu \mathrm{l}$ of dNTPs, $1 \mu \mathrm{l}$ of each primer, $9.2 \mu \mathrm{l}$ of double-distilled water and 100-500 ng of DNA template. The thermal cycling protocol followed Dissanayake et al. (2016a). The PCR products were verified by staining with ethidium bromide on $1.2 \%$ agarose electrophoresis gels and purified according to the manufacturer's instructions of Qiangen purification kit (Qiagen, Hilden, Germany). DNA sequencing of the genes were conducted by Sunbiotech Company, Beijing, China.

The systemic placement of taxa in this paper was based on the phylogeny of combined analysis of ITS and TEF (Phillips et al. 2013, Dissanayake et al. 2016b). DNASTAR V.5.1 and SEQMAN V.5.00 were used to obtain consensus sequences from sequences generated from forward and reverse primers. Sequences obtained from this study and other sequences originating from GenBank (Table 2) were aligned using default settings of MAFFT v.7 (Katoh \& Toh 2008, http://mafft.cbrc.jp/alignment/server/) and manually adjusted using BIOEDIT V.7.0.9.0 (Hall 1999), where necessary. Maximum-parsimony (MP) analysis was performed using PAUP v. $4.0 \mathrm{~b} 10$ (Swofford 2003) to obtain the most parsimonious trees. Gaps were treated as missing data. Trees were inferred using the heuristic search option with Tree Bisection Reconnection (TBR) branch swapping and 1000 random sequence additions. Maxtrees were set up to 1000 and branches of zero length were collapsed and all multiple parsimonious trees were saved. Descriptive tree statistics for parsimony such as Tree Length (TL), Consistency Index (CI), Retention Index (RI), Relative Consistency Index (RC) and Homoplasy Index (HI) were calculated. Clade stability was evaluated by 1000 bootstrap replications resulting from maximum parsimony analysis (Hillis \& Bull 1993). The best model of evolution for each gene region was determined by using MrModeltest 2.2 (Nylander 2004). The general time-reversible model of evolution (Rodriguez et al. 1990), including estimation of invariable sites and assuming a discrete gamma distribution with six rate categories $(\mathrm{GTR}+\mathrm{I}+\mathrm{G})$ was used for BI analyses for ITS and EF1- $\alpha$ sequence datasets. Posterior probabilities (PP) were determined by Markov Chain Monte Carlo sampling (BMCMC) in MrBayes v. 3.0b4 (Ronquist \& Huelsenbeck 2003). Four simultaneous Markov chains were run for 1000000 generations and trees were sampled every 100th generation and 10000 trees were obtained. The first 2000 trees, representing the burn-in phase of the analyses, were discarded while remaining 8000 trees were used for calculating posterior probabilities in the majority rule consensus tree (critical value for the topological convergence diagnostic set to 0.01) (Crous et al. 2006). Phylogenetic trees were drawn using Treeview v. 1.6.6 (Page 1996). The DNA sequences generated in this study are deposited in GenBank (Table 1) and the alignments in TreeBase (http://purl.org/phylo/treebase/phylows/study/). Taxonomic novelties were submitted to the Faces of Fungi database (Jayasiri et al. 2015) and Index Fungorum (Index Fungorum 2017).

Table 2 Isolates from GenBank used in phylogenetic analyses. Ex-type species are in bold.

\begin{tabular}{llll}
\hline Species & Isolate number & ITS & TEF \\
\hline Botryosphaeria agaves & MFLUCC 11-0125 & JX646791 & JX646856
\end{tabular}




\begin{tabular}{|c|c|c|c|}
\hline Species & Isolate number & ITS & TEF \\
\hline Botryosphaeria agaves & MFLUCC 10-0051 & JX646790 & JX646855 \\
\hline Botryosphaeria auasmontanum & CMW 25413 & KF766167 & EU101348 \\
\hline Botryosphaeria corticis & CBS 119047 & DQ299245 & EU017539 \\
\hline Botryosphaeria corticis & ATCC 22927 & DQ299247 & EU673291 \\
\hline Botryosphaeria dothidea & CBS 115476 & AY236949 & AY236898 \\
\hline Botryosphaeria dothidea & CBS 110302 & AY259092 & AY573218 \\
\hline Botryosphaeria fabicerciana & CBS 127193 & HQ332197 & HQ332213 \\
\hline Botryosphaeria fabicerciana & CMW 27108 & HQ332200 & HQ332216 \\
\hline Botryosphaeria fusispora & MFLUCC 10-0098 & JX646789 & JX646854 \\
\hline Botryosphaeria fusispora & MFLUCC 11-0507 & JX646788 & JX646853 \\
\hline Botryosphaeria minutispermatia & GZCC 16-0013 & KX447675 & KX447678 \\
\hline Botryosphaeria minutispermatia & GZCC 16-0014 & KX447676 & KX447679 \\
\hline Botryosphaeria ramosa & CBS 122069 & EU144055 & EU144070 \\
\hline Botryosphaeria scharifii & CBS 124703 & JQ772020 & JQ772057 \\
\hline Botryosphaeria scharifii & CBS 124702 & JQ772019 & JQ772056 \\
\hline Botryosphaeria sinensia & CGMCC 3.17722 & KT343254 & KU221233 \\
\hline Botryosphaeria sinensia & CGMCC 3.17724 & KT343256 & KU221234 \\
\hline Diplodia africana & CBS 120835 & EF445343 & EF445382 \\
\hline Diplodia africana & CBS 121104 & EF445344 & EF445383 \\
\hline Diplodia agrifolia & CBS 132777 & JN693507 & JQ517317 \\
\hline Diplodia agrifolia & UCROK1429 & JQ411412 & JQ512121 \\
\hline Diplodia alatafructa & CBS 124931 & FJ888460 & FJ888444 \\
\hline Diplodia alatafructa & CBS 124933 & FJ888478 & FJ888446 \\
\hline Diplodia allocellula & CBS 130408 & JQ239397 & JQ239384 \\
\hline Diplodia allocellula & CBS 130410 & JQ239399 & JQ239386 \\
\hline Diplodia bulgarica & CBS 124254 & GQ923853 & GQ923821 \\
\hline Diplodia bulgarica & CBS 124135 & GQ923852 & GQ923820 \\
\hline Diplodia corticola & CBS 112549 & AY259100 & AY573227 \\
\hline Diplodia corticola & CBS 112546 & AY259110 & DQ458872 \\
\hline Diplodia crataegicola & MFLU 15-1311 & KT290244 & KT290248 \\
\hline Diplodia cupressi & CBS 168.87 & DQ458893 & DQ458878 \\
\hline Diplodia cupressi & CBS 261.85 & DQ458894 & DQ458879 \\
\hline Diplodia estuarina & CMW41231 & KP860831 & KP860676 \\
\hline Diplodia estuarina & CMW41230 & KP860830 & KP860675 \\
\hline Diplodia fraxinii & CBS 136010 & KF307700 & KF318747 \\
\hline Diplodia galiicola & MFLU 15-1310 & KT290245 & KT290249 \\
\hline Diplodia insularis & CBS 140350 & KX833072 & KX833073 \\
\hline Diplodia intermedia & CBS 124462 & GQ923858 & GQ923826 \\
\hline Diplodia intermedia & CBS 124134 & HM036528 & GQ923851 \\
\hline Diplodia malorum & CBS 124130 & GQ923865 & GQ923833 \\
\hline Diplodia malorum & CBS 112554 & AY259095 & DQ458870 \\
\hline Diplodia mutila & CBS 112553 & AY259093 & AY573219 \\
\hline Diplodia mutila & CBS 230.30 & DQ458886 & DQ458869 \\
\hline Diplodia neojuniperi & CPC 22753 & KM006431 & KM006462 \\
\hline Diplodia olivarum & CBS 121887 & EU392302 & EU392279 \\
\hline Diplodia olivarum & CBS 121886 & EU392297 & EU392274 \\
\hline Diplodia pseudoseriata & CBS 124906 & EU080927 & EU863181 \\
\hline Diplodia pseudoseriata & CBS 124907 & EU080922 & EU863179 \\
\hline Diplodia quercivora & CBS 133852 & JX894205 & JX894229 \\
\hline Diplodia rosacearum & CBS 141915 & KT956270 & KU378605 \\
\hline Diplodia rosulata & CBS 116470 & EU430265 & EU430267 \\
\hline Diplodia rosulata & CBS 116472 & EU430266 & EU430268 \\
\hline Diplodia sapinea & CBS 393.84 & DQ458895 & DQ458880 \\
\hline Diplodia sapinea & CBS 109725 & DQ458896 & DQ458881 \\
\hline
\end{tabular}




\begin{tabular}{|c|c|c|c|}
\hline Species & Isolate number & ITS & TEF \\
\hline Diplodia scrobiculata & CBS 118110 & AY253292 & AY624253 \\
\hline Diplodia scrobiculata & CBS 109944 & DQ458899 & DQ458884 \\
\hline Diplodia scrobiculata & CBS 113423 & DQ458900 & DQ458885 \\
\hline Diplodia seriata & CBS 112555 & AY259094 & AY573220 \\
\hline Diplodia seriata & CBS 119049 & DQ458889 & DQ458874 \\
\hline Diplodia subglobosa & CBS 124133 & GQ923856 & GQ923824 \\
\hline Diplodia tsugae & CBS 418.64 & DQ458888 & DQ458873 \\
\hline Dothiorella acacicola & CBS 141295 & KX228269 & KX228376 \\
\hline Dothiorella americana & CBS 128309 & HQ288218 & HQ288262 \\
\hline Dothiorella americana & CBS 128310 & HQ288219 & HQ288263 \\
\hline Dothiorella brevicollis & CBS 130411 & JQ239403 & JQ239390 \\
\hline Dothiorella californica & CBS 141587 & KX357188 & KX357211 \\
\hline Dothiorella capri-amissi & CMW 25403 & EU101323 & EU101368 \\
\hline Dothiorella capri-amissi & CMW 25404 & EU101324 & EU101369 \\
\hline Dothiorella casuarini & CBS 120688 & DQ846773 & DQ875331 \\
\hline Dothiorella casuarini & CBS 120690 & DQ846774 & DQ875333 \\
\hline Dothiorella dulcispinae & CBS 130413 & JQ239400 & JQ239387 \\
\hline Dothiorella iberica & CBS 115041 & AY573202 & AY573222 \\
\hline Dothiorella iberica & CBS 113188 & AY573198 & EU673278 \\
\hline Dothiorella iranica & IRAN1587C & KC898231 & KC898214 \\
\hline Dothiorella longicollis & CBS 122068 & EU144054 & EU144069 \\
\hline Dothiorella longicollis & CBS 122066 & EU144052 & EU144067 \\
\hline Dothiorella moneti & MUCC505 & EF591920 & EF591971 \\
\hline Dothiorella moneti & MUCC 507 & EF591922 & EF591973 \\
\hline Dothiorella neclivorem & DAR80992 & KJ573643 & KJ5573640 \\
\hline Dothiorella oblonga & CMW 25407 & EU101300 & EU101345 \\
\hline Dothiorella omnivora & CBS 140349 & KP205497 & KP205470 \\
\hline Dothiorella omnivora & CBS 188.87 & EU673316 & EU673283 \\
\hline Dothiorella parva & IRAN1579C & КC898234 & КC898217 \\
\hline Dothiorella parva & IRAN1585C & KC898235 & KC898218 \\
\hline Dothiorella pretoriensis & CBS 130404 & JQ239405 & JQ239392 \\
\hline Dothiorella pretoriensis & CBS 130403 & JQ239406 & JQ239393 \\
\hline Dothiorella prunicola & CBS 124723 & EU673313 & EU673280 \\
\hline Dothiorella rhamni & MFLUCC 14-0902 & KU246381 & N/A \\
\hline Dothiorella santali & MUCC 509 & EF591924 & EF591975 \\
\hline Dothiorella santali & MUCC 508 & EF591923 & EF591974 \\
\hline Dothiorella sarmentorum & IMI 63581b & AY573212 & AY573235 \\
\hline Dothiorella sarmentorum & CBS 115038 & AY573206 & AY573223 \\
\hline Dothiorella sempervirentis & IRAN1583C & KC898236 & КC898219 \\
\hline Dothiorella sempervirentis & IRAN1581C & KC898237 & KC898220 \\
\hline Dothiorella striata & ICMP16824 & EU673320 & EU673287 \\
\hline Dothiorella striata & ICMP16819 & EU673320 & EU673287 \\
\hline Dothiorella symphoricarposicola & MFULCC 13-0497 & KJ742378 & KJ742381 \\
\hline Dothiorella symphoricarposicola & MFLUCC 13-0196 & KU234782 & KU234796 \\
\hline Dothiorella tectonae & MFLUCC12-0382 & KM396899 & KM409637 \\
\hline Dothiorella thailandica & CBS 133991 & JX646796 & JX646861 \\
\hline Dothiorella thripsita & BRIP 51876 & FJ824738 & KJ573639 \\
\hline Dothiorella ulmacea & CBS 138855 & KR611881 & KR611910 \\
\hline Dothiorella uruguayensis & CBS 124908 & EU080923 & EU863180 \\
\hline Dothiorella vidmadera & DAR78992 & EU768874 & EU768881 \\
\hline Dothiorella vidmadera & DAR78993 & EU768876 & EU768882 \\
\hline Dothiorella vinea-gemmae & DAR81012 & KJ573644 & KJ573641 \\
\hline Eutiarosporella africana & CBS 133854 & KC769956 & KC769852 \\
\hline
\end{tabular}




\begin{tabular}{|c|c|c|c|}
\hline Species & Isolate number & ITS & TEF \\
\hline Eutiarosporella africana & CBS 135850 & KC769957 & KC769853 \\
\hline Eutiarosporella dactylidis & MFLUCC 13-0276 & KM978944 & KP031694 \\
\hline Eutiarosporella dactylidis & MFLUCC 15-0915 & KU246378 & N/A \\
\hline Eutiarosporella darliae & DAR 82491 & KP309793 & KP309805 \\
\hline Eutiarosporella darliae & DAR 82493 & KP309786 & KP309807 \\
\hline Eutiarosporella pseudodarliae & DAR 82489 & KP309796 & KP309797 \\
\hline Eutiarosporella pseudodarliae & DAR 82490 & КР309794 & KP309800 \\
\hline Eutiarosporella tritici & CBS 118719 & KC769961 & KF531809 \\
\hline Eutiarosporella tritici-australis & DAR 82485 & KP309788 & KP309799 \\
\hline Eutiarosporella tritici-australis & DAR 82486 & KP309787 & KP309804 \\
\hline Eutiarosporella urbis-rosarum & CBS 130405 & JQ239407 & JQ239394 \\
\hline Eutiarosporella urbis-rosarum & CBS 130406 & JQ239408 & JQ239395 \\
\hline Neofusicoccum algeriense & CBS 137504 & KJ657702 & KJ657715 \\
\hline Neofusicoccum algeriense & ALG9 & KJ657704 & KJ657721 \\
\hline Neofusicoccum andinum & CBS 117453 & AY693976 & AY693977 \\
\hline Neofusicoccum andinum & CBS 117452 & DQ306263 & DQ306264 \\
\hline Neofusicoccum arbuti & CBS 116131 & AY819720 & N/A \\
\hline Neofusicoccum arbuti & CBS 117090 & AY819724 & KF531791 \\
\hline Neofusicoccum australe & CMW 6837 & AY339262 & AY339270 \\
\hline Neofusicoccum australe & CMW 6853 & AY339263 & AY339271 \\
\hline Neofusicoccum batangarum & CBS 124924 & FJ900607 & FJ900653 \\
\hline Neofusicoccum batangarum & CBS 124923 & FJ900608 & FJ900654 \\
\hline Neofusicoccum braziliense & CMM1338 & JX513630 & JX513610 \\
\hline Neofusicoccum cordaticola & CBS 123634 & EU821898 & EU821868 \\
\hline Neofusicoccum cordaticola & CBS 123635 & EU821903 & EU821873 \\
\hline Neofusicoccum cryptoaustrale & CMW23785 & FJ752742 & FJ752713 \\
\hline Neofusicoccum eucalypticola & CBS 115679 & AY615141 & AY615133 \\
\hline Neofusicoccum eucalypticola & CBS 115766 & AY615143 & AY615135 \\
\hline Neofusicoccum eucalyptorum & CBS 115791 & AF283686 & AY236891 \\
\hline Neofusicoccum eucalyptorum & CMW 10126 & AF283687 & AY236892 \\
\hline Neofusicoccum grevilleae & CBS 129518 & JF951137 & N/A \\
\hline Neofusicoccum hellenicum & CERC1947 & KP217053 & KP217061 \\
\hline Neofusicoccum kwambonambiense & CBS 123639 & EU821900 & EU821870 \\
\hline Neofusicoccum kwambonambiense & CBS 123641 & EU821919 & EU821889 \\
\hline Neofusicoccum lumnitzerae & CMW41469 & KP860881 & KP860724 \\
\hline Neofusicoccum lumnitzerae & CMW41228 & KP860882 & KP860725 \\
\hline Neofusicoccum luteum & CBS 110299 & AY259091 & AY573217 \\
\hline Neofusicoccum luteum & CBS 110497 & EU673311 & EU673277 \\
\hline Neofusicoccum macroclavatum & CBS 118223 & DQ093196 & DQ093217 \\
\hline Neofusicoccum macroclavatum & WAC 12446 & DQ093197 & DQ093218 \\
\hline Neofusicoccum mangiferae & CBS 118531 & AY615185 & DQ093221 \\
\hline Neofusicoccum mangiferae & CBS 118532 & AY615186 & DQ093220 \\
\hline Neofusicoccum mangroviorum & CMW41365 & KP860859 & KP860702 \\
\hline Neofusicoccum mangroviorum & CMW42481 & KР860848 & KP860692 \\
\hline Neofusicoccum mediterraneum & CBS 121718 & GU251176 & GU251308 \\
\hline Neofusicoccum mediterraneum & CBS 121558 & GU799463 & GU799462 \\
\hline Neofusicoccum nonquaesitum & CBS 126655 & GU251163 & GU251295 \\
\hline Neofusicoccum nonquaesitum & PD 301 & GU251164 & GU251296 \\
\hline Neofusicoccum occulatum & CBS 128008 & EU301030 & EU339509 \\
\hline Neofusicoccum occulatum & MUCC 286 & EU736947 & EU339511 \\
\hline Neofusicoccum parvum & CMW 9081 & AY236943 & AY236888 \\
\hline Neofusicoccum parvum & CBS 110301 & AY259098 & AY573221 \\
\hline Neofusicoccum pennatisporum & MUCC 510 & EF591925 & EF591976 \\
\hline
\end{tabular}




\begin{tabular}{llll}
\hline Species & Isolate number & ITS & TEF \\
\hline Neofusicoccum protearum & STE-U 4361 & AF196295 & N/A \\
Neofusicoccum protearum & STE-U 1775 & AF452539 & N/A \\
Neofusicoccum ribis & CBS 115475 & AY236935 & AY236877 \\
Neofusicoccum ribis & CBS 121.26 & AF241177 & AY236879 \\
Neofusicoccum umdonicola & CBS 123645 & EU821904 & EU821874 \\
Neofusicoccum umdonicola & CBS 123646 & EU821905 & EU821875 \\
Neofusicoccum ursorum & CMW 24480 & FJ752746 & FJ752709 \\
Neofusicoccum viticlavatum & CBS 112878 & AY343381 & AY343342 \\
Neofusicoccum viticlavatum & CBS 112977 & AY3433380 & AY343341 \\
Neofusicoccum vitifusiforme & CBS 110887 & AY343383 & AY343343 \\
Neofusicoccum vitifusiforme & CBS 110880 & AY3433382 & AY343344 \\
\hline
\end{tabular}

\section{Results}

\section{Sample collection}

In this study, a collection of 52 saprobic specimens from different hosts in urban and forest tree species were collected and this resulted in identification of 14 Botryosphaeriaceae species. Of these, 12 isolates, each from B. dothidea (23\%) and Di. seriata $(23 \%)$ were detected as the prominent species. Six isolates of the newly described Do. italica $(12 \%)$ were obtained, while all other species were in low numbers of isolates. Host ranges of the species were determined from available literature and as well as from the SMML Fungus-Host Distribution Database. Several new fungus-host associations were established (Table 1).

\section{Phylogenetic analyses}

In total, five multi-gene phylogenies were generated (Figs 1-5). With the support values of Bayesian analyses, maximum parsimony trees from each alignment are presented here. The analyses treated Botryosphaeria (Fig. 1), Diplodia (Fig. 2), Dothiorella (including Spencermartinsia, Fig. 3), Eutiarosporella (Fig. 4) and Neofusicoccum (Fig. 5). PCR amplifications of the ITS and TEF regions gave products of 0.5 and $0.4 \mathrm{~kb}$, respectively.

The combined ITS and TEF data set of Botryosphaeria consists of 33 strains with Macrophomina phaseolina (CBS 227.33) as the outgroup taxon. The best scoring parsimony tree is shown in Fig. 1 and revealed two known species: B. dothidea and B. auasmontanum F.J.J. Van der Walt, Slippers \& G.J. Marais. The combined ITS and EF1- $\alpha$ dataset consisted of 824 characters (including alignment gaps). Of those, 667 were constant and 79 were variable and parsimony uninformative. Maximum parsimony analysis of the remaining 78 parsimony informative characters resulted in 199 equally parsimonious trees $(\mathrm{CI}=0.894, \mathrm{RI}=0.864, \mathrm{RC}=0.772, \mathrm{HI}=$ 0.106). Alignment and trees were deposited in TreeBASE (S21254). The phylogenetic analysis of Diplodia revealed five known species Di. alatafructa J.W.M. Mehl \& B. Slippers, Di. crataegicola Dissanayake, Camporesi \& K.D. Hyde, Di. mutila, Di. sapinea (Fr.) Fuckel and Di. seriata. The combined ITS and EF1- $\alpha$ dataset consisted of 839 characters (including alignment gaps) for 64 ingroup and 1 outgroup taxa. Of the 839 characters 611 were constant and 87 were variable and parsimony uninformative. Maximum parsimony analysis of the remaining 141 parsimony informative characters resulted in 398 equally parsimonious trees $(\mathrm{CI}=0.719, \mathrm{RI}=0.885, \mathrm{RC}=0.49$ $0.6375, \mathrm{HI}=0.0 .281$ ) and the best scoring first MP tree is shown in Fig. 2. Alignment and trees were deposited in TreeBASE (S21255).The Dothiorella phylogeny revealed four known species Do. omnivora, Do. rhamni Wanasinghe, Bulgakov, E.B.G. Jones \& K.D. Hyde, Do. sarmentorum, Do. sempervirentis Abdollahz., Zare \& A.J.L. Phillips and one taxonomic novelty which is treated below. The combined ITS and EF1- $\alpha$ dataset consisted of 737 characters (including alignment gaps) for 74 ingroup and 1 outgroup taxa. Of these, 484 were constant and 80 were variable and parsimony uninformative. Maximum parsimony analysis of the remaining 173 parsimony informative characters resulted in 644 equally parsimonious trees $(\mathrm{CI}=0.564, \mathrm{RI}=0.832, \mathrm{RC}=$ $0.469, \mathrm{HI}=0.436$ ) and the best scoring first MP tree is shown in Fig. 3. Alignment and trees were 
deposited in TreeBASE (S21256). The Eutiarosporella phylogeny discovered only one known species E. dactylidis (Thambug., Camporesi \& K.D. Hyde) Dissanayake, Camporesi \& K.D. Hyde. The combined ITS and EF1- $\alpha$ dataset consisted of 735 characters (including alignment gaps) for 17 ingroup and 1 outgroup taxa. Of the 735 characters 601 were constant and 601 were variable and parsimony uninformative. Maximum parsimony analysis of the remaining 72 parsimony informative characters resulted in six equally parsimonious trees $(\mathrm{CI}=0.853, \mathrm{RI}=0.861, \mathrm{RC}=$ $0.735, \mathrm{HI}=0.147$ ) and the best scoring first MP tree is shown in Fig. 4. Alignment and trees were deposited in TreeBASE (S21257). Phylogenetic analysis of Neofusicoccum revealed only one known species $N$. parvum. The combined ITS and EF1- $\alpha$ dataset consisted of 817 characters (including alignment gaps) for 55 ingroup and 1 outgroup taxa. Of these characters 600 were constant and 89 were variable and parsimony uninformative. Maximum parsimony analysis of the remaining 128 parsimony informative characters resulted in 44 equally parsimonious trees $(\mathrm{CI}=$ 0.717, $\mathrm{RI}=0.878, \mathrm{RC}=0.629, \mathrm{HI}=0.283$ ) and the best scoring first $\mathrm{MP}$ tree is shown in Fig. 5. Alignment and trees were deposited in TreeBASE (S21258).

\section{Taxonomy}

Dothiorella italica Dissanayake, Camporesi \& K.D. Hyde, sp. nov.

Index Fungorum number: IF553566; Facesoffungi number: FoF03426; Fig. 6

Etymology - Referring to the locality where the specimen was collected.

Holotype - MFLU 17-0290.

Saprobic on dead aerial branch of Rosa canina (Rosaceae). Sexual morph: Undetermined. Asexual morph: Conidiomata up to $680 \mu \mathrm{m}$ wide, globose, pycnidial, stromatic, solitary, composed of dark brown thick-walled textura angularis. Ostiolate. Ostiole up to $65 \mu \mathrm{m}$ in height. Conidiogenous cells 8-15 $\times 3-6 \mu \mathrm{m}$, lining the pycnidial cavity, holoblastic, hyaline, subcylindrical. Conidia $28.7-43.2 \times 13.2-17.5 \mu \mathrm{m}(35.7 \times 15.6 \mu \mathrm{m}, \mathrm{n}=20)$ initially hyaline and aseptate becoming pigmented brown and 1-septate often while still attached to conidiogenous cell, slightly constricted at the septum, ovoid with a broadly rounded apex and truncate base.

Culture characteristics - Colonies on PDA, covering entire petri dishes after $12 \mathrm{~d}$ in the dark at $25^{\circ} \mathrm{C}$; circular, initially white, after $7 \mathrm{~d}$ becoming greyish brown to black; reverse grey to dark grayish green.

Material examined - ITALY. Province of Forlì-Cesena [FC], near Strada San Zeno Galeata, on dead aerial branch of Rosa canina (Rosaceae), 17 October 2014, Erio Camporesi IT 2179 (MFLU 17-0290, holotype), ex-type living culture MFLUCC 17-0951. Details of studied additional materials are given in Table 1.

Notes - Based on multigene phylogenetic analyses, six isolates of Do. italica formed a clade close to Do. prunicola A.J.L. Phillips \& Abdollahz. The novel species differs from Do. prunicola by 16 nucleotides in the concatenated alignment, in which five were distinct in the ITS region and eleven in the TEF region. Morphologically, the length of conidia of Do. prunicola are smaller (19$30.5 \mu \mathrm{m})$ compared to those of Do. italica $(28.7-43.2 \mu \mathrm{m})$.

\section{Discussion}

Studies on Botryosphaeriaceae, dealing with the phylogenetic traits and morphology of isolates associated with various hosts, have increased in recent years, enabling the worldwide identification of taxa at the species level (Liu et al. 2012, Phillips et al. 2013, Dissanayake et al. 2016b). The current study provides details from sampling over the past five years which has led to characterization of 14 species in Botryosphaeriaceae, especially in the provinces of Forlì-Cesena, Arezzo and Perugia in Italy. Of these, Dothiorella italica is illustrated and described as a novel species. Botryosphaeria auasmontanum (Slippers et al. 2014), Di. alatafructa (Mehl et al. 2011) and Do. sempervirentis (Abdollahzadeh et al. 2014) were reported as new records for Italy. However, when considering the phylogenetic analysis of Botryosphaeria species (Fig. 1), B. dothidea/B. auasmontanum appear to be synonyms. 


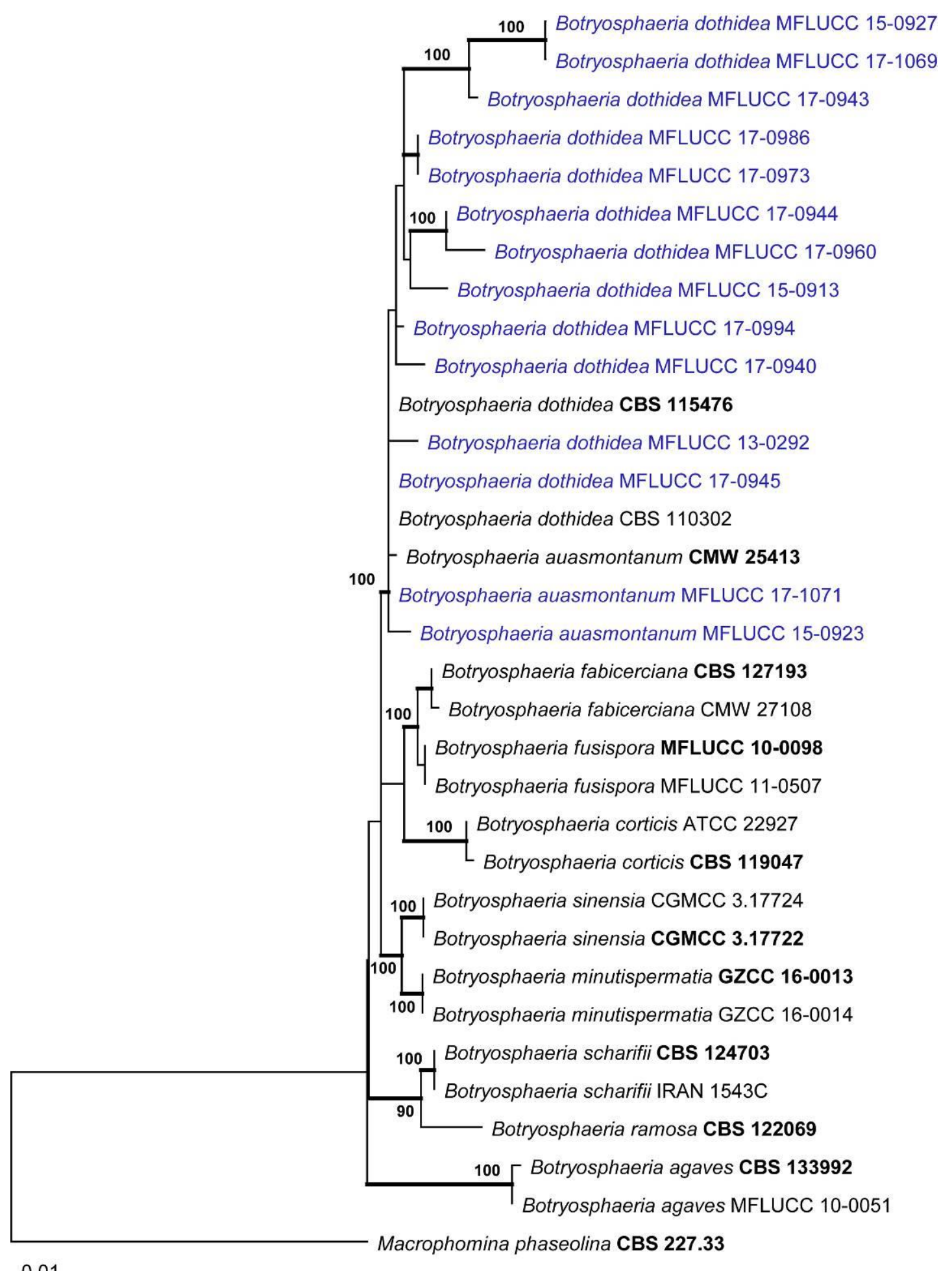

0.01

Figure 1 - Phylogram generated from maximum parsimony analysis of Botryosphaeria species based on combined ITS and TEF sequence data. Parsimony bootstrap support values for MP $\geq 90 \%$ are indicated above the nodes and the branches are in bold indicate Bayesian posterior probabilities $\geq 0.9$. The tree is rooted with Macrophomina phaseolina (CBS 227.33). Isolate numbers of ex-types and reference strains are in bold. Species isolated in this study are in blue. 


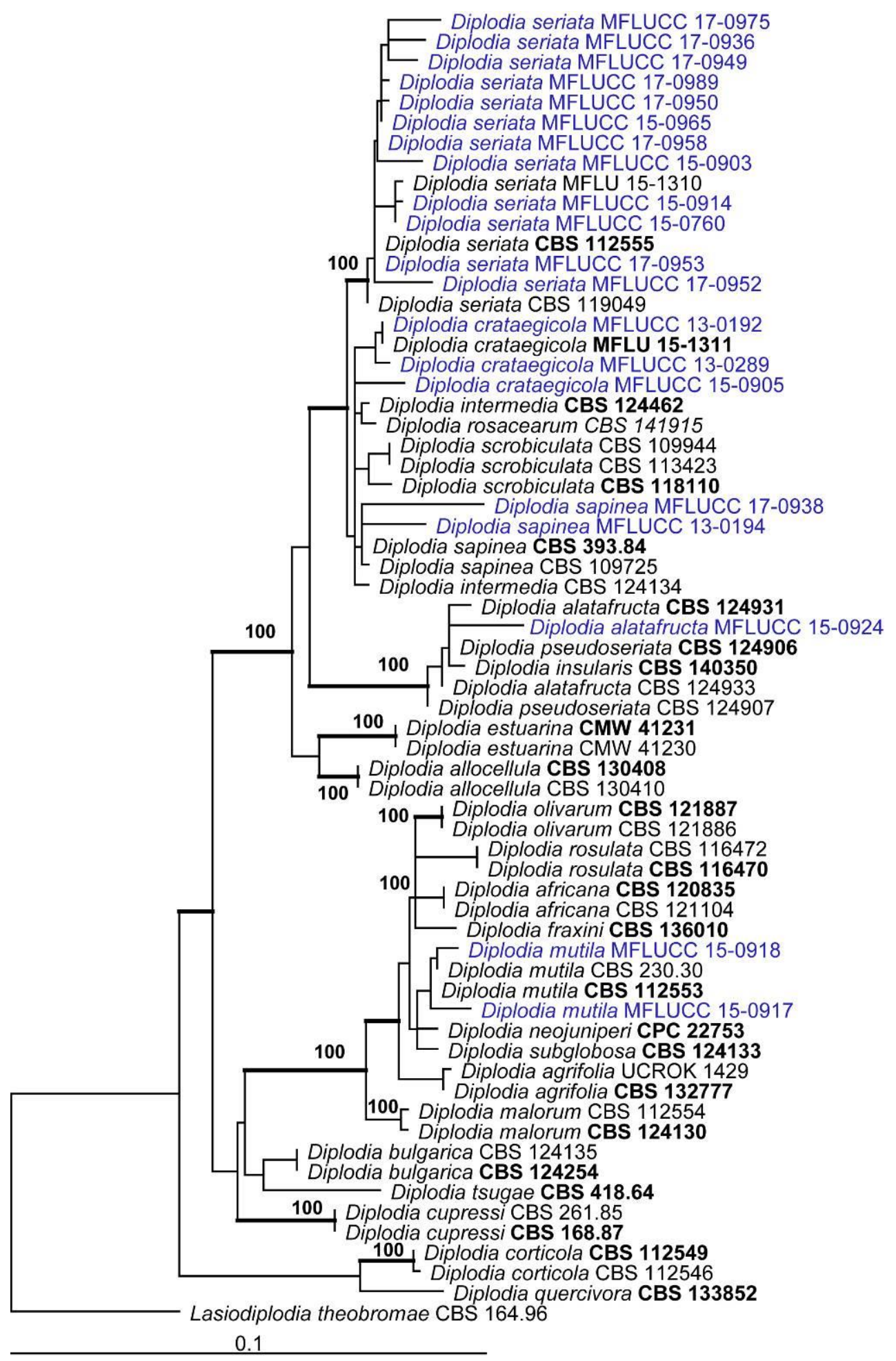

Figure 2 - Phylogram generated from maximum parsimony analysis of Diplodia species based on combined ITS and TEF sequence data. Parsimony bootstrap support values for MP $\geq 90 \%$ are indicated above the nodes and the branches are in bold indicate Bayesian posterior probabilities $\geq 0.9$. The tree is rooted with Lasiodiplodia theobromae (CBS 164.96). Isolate numbers of ex-types and reference strains are in bold. Species isolated in this study are in blue. 


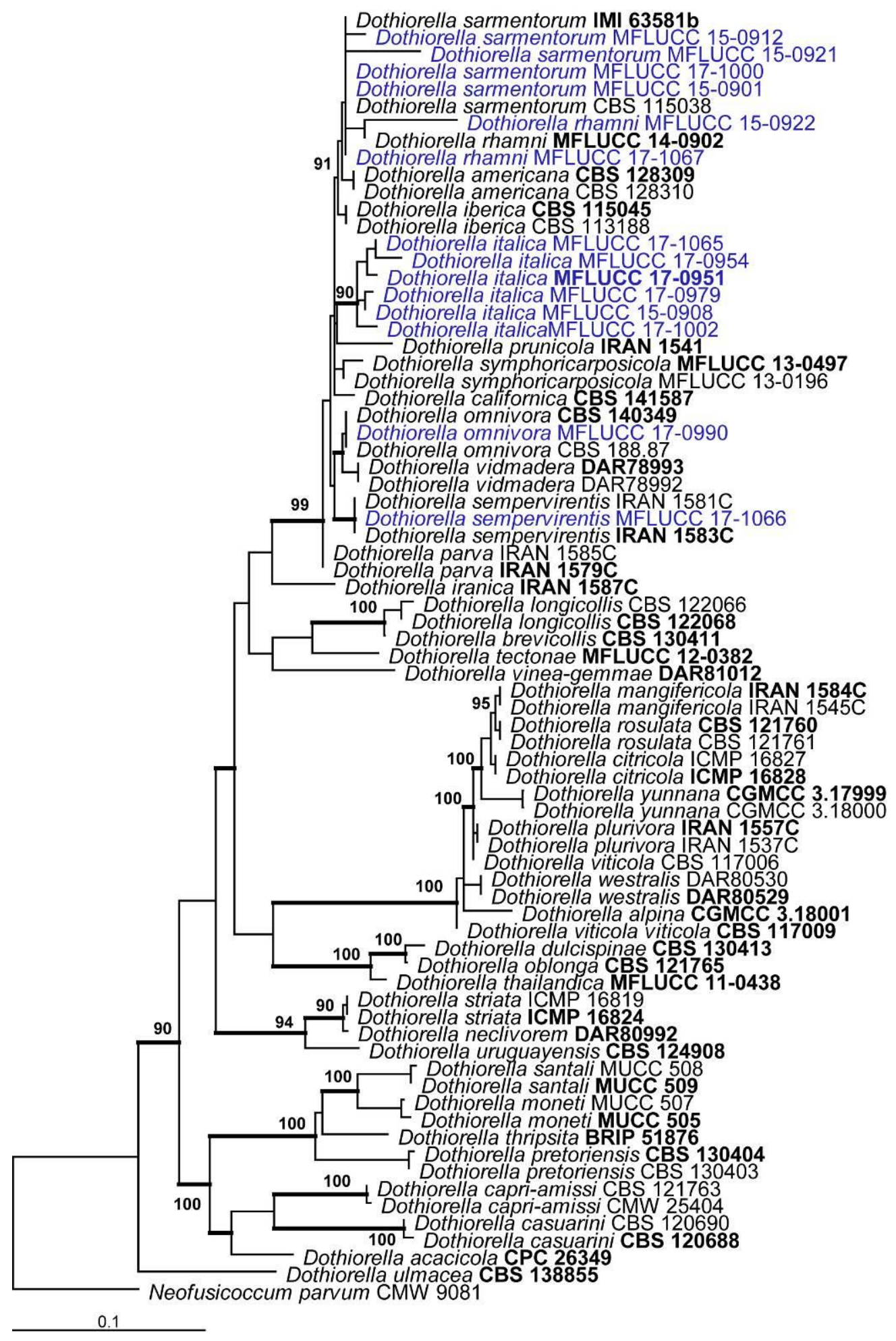

Figure 3 - Phylogram generated from maximum parsimony analysis of Dothiorella species based on combined ITS and TEF sequence data. Parsimony bootstrap support values for MP $\geq 90 \%$ are indicated above the nodes and the branches are in bold indicate Bayesian posterior probabilities $\geq 0.9$. The tree is rooted with Neofusicoccum parvum (CMW 9081). Isolate numbers of ex-types and reference strains are in bold. Species isolated in this study are in blue. 


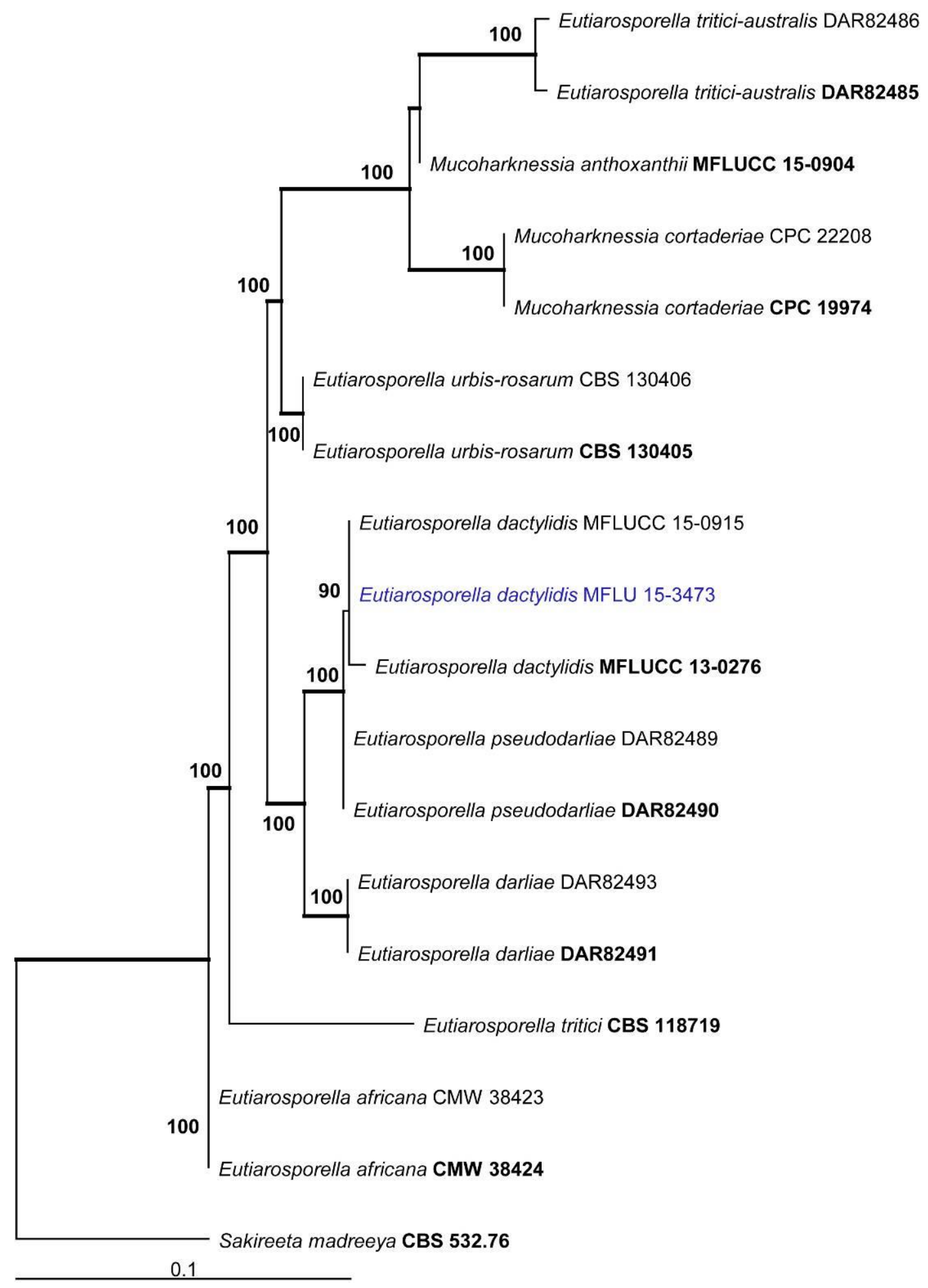

Figure 4 - Phylogram generated from maximum parsimony analysis of Eutiarosporella species based on combined ITS and TEF sequence data. Parsimony bootstrap support values for MP $\geq 90 \%$ are indicated above the nodes and the branches are in bold indicate Bayesian posterior probabilities $\geq 0.9$. The tree is rooted with Sakireeta madreeya (CBS 532.76). Isolate numbers of ex-types and reference strains are in bold. Species isolated in this study are in blue. 


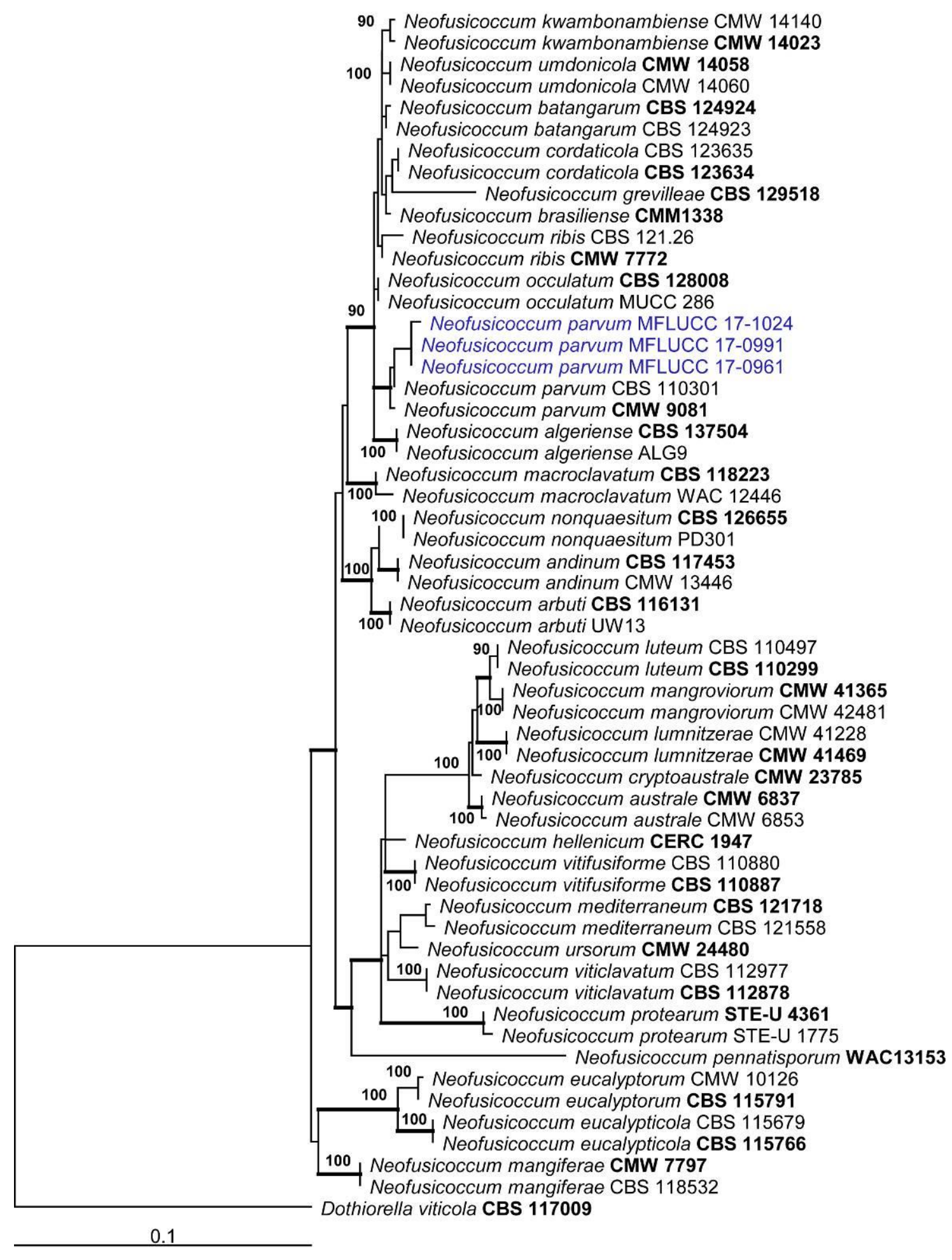

Figure 5 - Phylogram generated from maximum parsimony analysis of Neofusicoccum species based on combined ITS and TEF sequence data. Parsimony bootstrap support values for MP $\geq 90 \%$ are indicated above the nodes and the branches are in bold indicate Bayesian posterior probabilities $\geq 0.9$. The tree is rooted with Dothiorella viticola (CBS 117009). Isolate numbers of ex-types and reference strains are in bold. Species isolated in this study are in blue. 


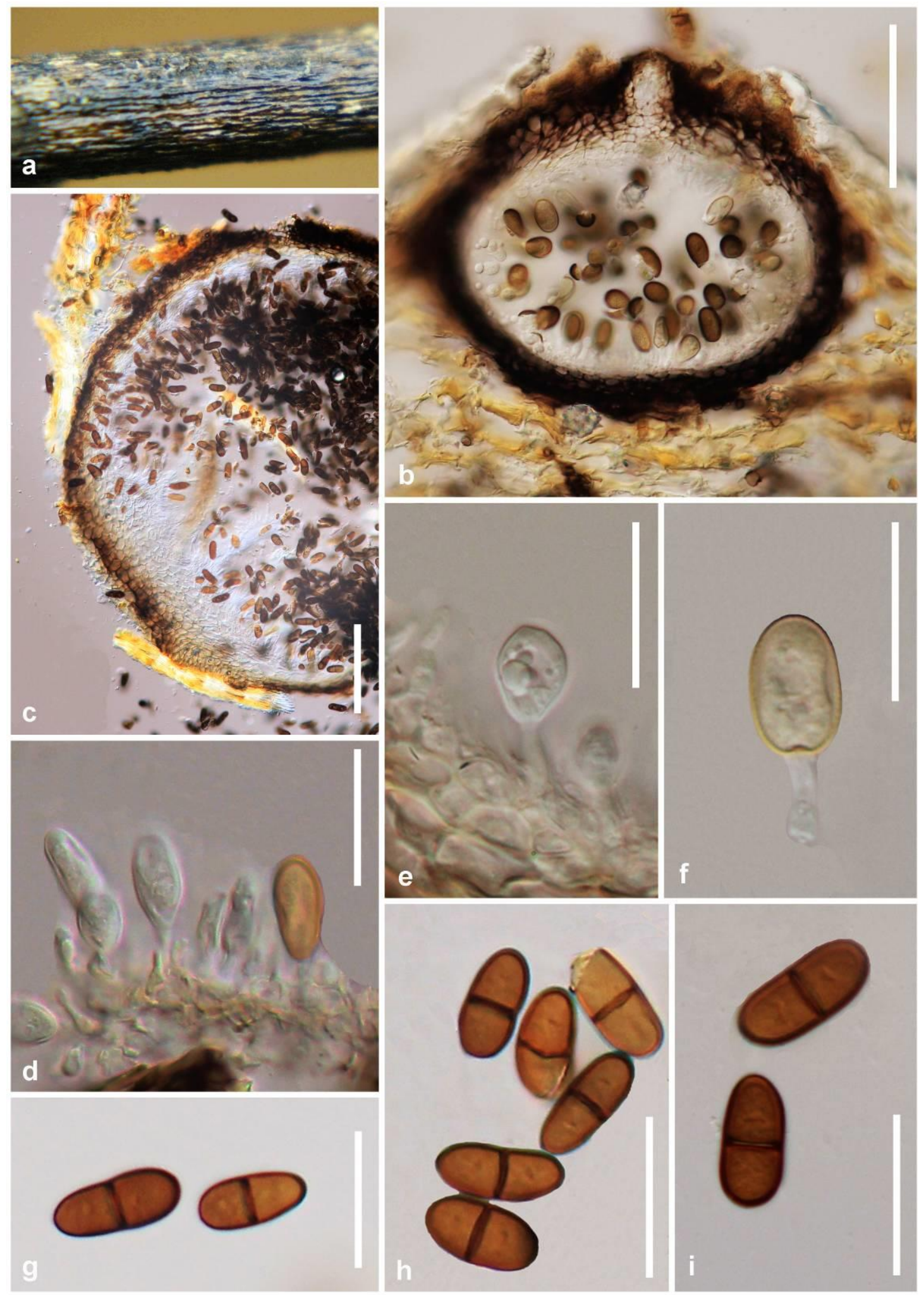

Fig. 6 Dothiorella italica (MFLU 17-0290). a - Conidiomata on host surface, b - Cross section of conidioma, $\mathbf{c}-$ Peridium, $\mathbf{d}-\mathbf{e}$ Immature and mature conidia attached to conidiogenous cells. $\mathbf{g}-\mathbf{i}$ Mature 1-septate brown conidia. Scale bars: $b, c=200 \mu \mathrm{m}, \mathrm{d}-\mathrm{i}=40 \mu \mathrm{m}$.

Hence we suggest a careful comparison of these two species morphologically and at the molecular level to clarify the possible synonymy of $B$. auasmontanum under B. dothidea. Ten species, Botryosphaeria dothidea, Diplodia crataegicola, Di. mutila, Di. sapinea, Di. seriata, Dothiorella omnivora, Do. rhamni, Do. sarmentorum, Eutiarosporella dactylidis and 
Neofusicoccum parvum have already been reported from Italy (Dissanayake et al. 2016a, b). In this study, B. dothidea from Galium sp. (Rubiaceae), Carpinus betulus (Betulaceae), Laburnum anagyroides (Fabaceae), Colutea arborescens (Fabaceae) and Euonymus europaeus (Celastraceae); Di. crataegicola from Prunus sp. (Rosaceae) and Tilia sp. (Malvaceae); Di. mutila from Acer negundo (Sapindaceae) and Colutea arborescens (Fabaceae); Do. omnivora from Cornus sanguinea (Cornaceae); Do. rhamni from Tamarix gallica (Tamaricaceae); Do. sarmentorum from Clematis vitalba (Ranunculaceae) and Robinia pseudacacia (Fabaceae); N. parvum from Eupatorium cannabinum (Asteraceae) are described as new host records based on SMML Fungus-Host Distribution Database.The gene regions commonly used to infer phylogenetic relationships within species in the Botryosphaeriaceae (ITS and TEF) made it potential to identify species with confidence.

Botryosphaeriaceae species in host plants in Italy vary in their abundance, distribution and host association (Giambra et al. 2016, Linaldeddu et al. 2016a). Botryosphaeria and Diplodia were the most frequently isolated genera in this study and this inference is consistent with several previous reports (Lazzizera et al. 2008, Linaldeddu et al. 2014, 2015, Giambra et al. 2016, Marsberg et al. 2017), which show the capability of species in these genera to occupy a wide range of plant species and geographic areas worldwide. Some Diplodia species have wide host ranges, for example Di. seriata which has been recorded on more than 250 hosts (Phillips et al. 2013, Dissanayake et al. 2016b). Botryosphaeria dothidea is one of the most common species on a large number of hosts and has been reported from hundreds of plant species with a broad global distribution (Phillips et al. 2013, Dissanayake et al. 2016b). Our study has confirmed this, by isolating B. dothidea from eleven host plant species (Table 1). Dothiorella species are endophytes, pathogens and saprobes of a wide range of woody hosts (Crous et al. 2006, Liu et al. 2012, Phillips et al. 2013, Hyde et al. 2013, 2014, Dissanayake et al. 2016a,). Although there are 390 species records for Dothiorella in Index fungorum (accessed in June 2017, http://www.indexfungorum.org), Dissanayake et al. (2016b) revealed that cultures are available for only 30 species. Among the species in this genus, Do. sarmentorum has a worldwide distribution associated with a variety of hosts (Gonzalez-Dominguez et al. 2016, Zlatkovic' et al. 2016). Our study revealed four Do. sarmentorum isolates from four different hosts. The newly described $D o$. italica species was isolated from Forlì-Cesena and Perugia provinces in Italy and all isolates were recovered from dead aerial branches of Cupressus sp. (Cupressaceae), Ligustrum sp. (Oleaceae), Melia azedarach (Meliaceae), Prunus sp. (Rosaceae), Rosa canina (Rosaceae) and Rubus sp. (Rosaceae). The isolation of this novel species from different host plants implies its wide distribution and indistinct host association. Diplodia sapinea is a well-known pathogen of Pinus spp. worldwide (Phillips et al. 2013). Two isolates of Di. sapinea obtained in this study from Picea excelsa (Pinaceae) and Pinus pinaster (Pinaceae) confirmed that, this species is more specific in Pinaceae hosts. Neofusicoccum parvum is a common and cosmopolitan species on diverse host plants and recognized as an aggressive pathogen of Actinidia sp., Juglans regia, Prunus laurocerasus, Vaccinium sp. and Vitis vinifera (Yan et al. 2013, Boyzo-Marin et al. 2016, Yu et al. 2016, Zlatkovic et al. 2016, Diaz et al. 2017). Our study revealed three saprobic N. parvum isolates from Eupatorium cannabinum (Asteraceae), Salix sp. (Salicaceae) and Vitis vinifera (Vitaceae).

Members of Botryosphaeriaceae family represent a growing threat to agricultural crops, urban and natural forest ecosystems in Italy (Linaldeddu et al. 2014, 2015, 2016a). This finding raises questions about the origin, introduction and pathway of these fungi as well as underlining the need to develop suitable actions to limit their further spread.

\section{Acknowledgement}

This work was financed by CARS-30 and JNKYT201605.

\section{References}


Abdollahzadeh J, Javadi A, Zare R, Phillips AJL. 2014 - A phylogenetic study of Dothiorella and Spencermatinsia species associated with woody plants in Iran, New Zealand, Portugal and Spain. Persoonia 32, 1-12.

Alves A, Correia A, Luque J, Phillips AJL. 2004 - Botryosphaeria corticola, sp. nov. on Quercus species, with notes and description of Botryosphaeria stevensii and its anamorph, Diplodia mutila. Mycologia 96, 598-613.

Barr ME. 1987 - Prodromus to class Class Loculoascomycetes. Published by the author, Amherst, Massachusetts, USA.

Boyzo-Marin J, Rebollar-Alviter A, Silva-Rojas HV, Ramirez-Maldonaldo G. 2016 - First report of Neofusicoccum parvum causing stem blight and dieback of blueberry in Mexico. Plant Disease https://doi.org/10.1094/PDIS-03-16-0381-PDN.

Carbone I, Kohn LM. 1999 - A method for designing primer sets for speciation studies in filamentous ascomycetes. Mycologia 91, 553-556.

Carlucci A, Cibelli F, Lops F, Raimondo ML. 2015 - Characterization of Botryosphaeriaceae species as causal agents of trunk disease on grapevines. Plant Disease 99, 1678-1688.

Chomnunti P, Hongsanan S, Hudson BA, Tian Q. et al. 2014 - The sooty moulds. Fungal Diversity $66,1-36$.

Crous PW, Müller MM, Sánchez RM, Giordano L, et al. 2015 - Resolving Tiarosporella spp. allied to Botryosphaeriaceae and Phacidiaceae. Phytotaxa 202, 073-093.

Crous PW, Slippers B, Wingfield MJ, Rheeder J. et al. 2006 - Phylogenetic lineages in the Botryosphaeriaceae. Studies in Mycology 55, 235-253.

Diaz GA, Latorre BA, Lolas M, Ferrada E. et al. 2017 - Identification and characterization of Diaporthe ambigua, D. australafricana, D. novem, and D. rudis causing a postharvest fruit rot in Kiwifruit. Plant Disease, https://doi.org/10.1094/PDIS-10-16-1535-RE.

Dissanayake AJ, Camporesi E, Hyde KD, Phillips AJL. et al. 2016a - Dothiorella species associated with woody hosts in Italy. Mycosphere 7, 51-63.

Dissanayake AJ, Phillips AJL, Hyde KD, Li XH. 2016b - Botryosphaeriaceae: Current status of genera and species. Mycosphere 7, 1001-1073.

Giambra S, Piazza G, Alves A, Mondello V, et al. 2016 - Botryosphaeriaceae species associated with diseased loquat trees in Italy and description of Diplodia rosacearum sp. nov. Mycosphere 7, 978-989.

Gonzalez-Domingueza E, Alves A, Leon M, Armengola J. 2016 - Characterization of Botryosphaeriaceae species associated with diseased loquat (Eriobotrya japonica) in Spain. Plant Pathology Doi: 10.1111/ppa.12556.

Hall TA. 1999 - BioEdit: a user-friendly biological sequence alignment editor and analysis program for Windows 95/98/NT. Nucleic Acids Symposium Series 41, 95-98.

Hillis DM, Bull JJ. 1993 - An empirical test of bootstrapping as a method for assessing confidence in phylogenetic analysis. Systamatic Biology 42, 182-192.

Hyde KD, Jones EBG, Liu JK, Ariyawansa H. et al. 2013 - Families of Dothideomycetes. Fungal Diversity 63, 1-313.

Hyde KD, Nilsson RH, Alias SA, Ariyawansa HA. et al. 2014 - One stop shop: backbones trees for important phytopathogenic genera: I. Fungal Diversity 67, 21-125.

Index Fungorum. 2017 - http://www.indexfungorum.org/Names/Names.asp.

Jami F, Slippers B, Wingfield MJ, Gryzenhout M. 2012 - Five new species of the Botryosphaeriaceae from Acacia karoo in South Africa. Cryptogamie Mycologie 33, 245266.

Jami F, Slippers B, Wingfield MJ, Gryzenhout M. 2014 - Botryosphaeriaceae species overlap on four unrelated, native South African hosts. Fungal Biology 118, 168-179.

Jayasiri SC, Hyde KD, Ariyawansa HA, Bhat J. et al. 2015 - The Faces of Fungi database: fungal names linked with morphology, phylogeny and human impacts. Fungal Diversity 74, 3-18.

Katoh K, Toh H. 2008 - Recent developments in the MAFFT multiple sequence alignment program. Briefings in Bioinformatics 9, 276-285. 
Lazzizera C, Frisullo S, Alves A, Phillips AJL. 2008 - Morphology, phylogeny and pathogenicity of Botryosphaeria and Neofusicoccum species associated with drupe rot of olives in southern Italy. Plant Pathology 57, 948-956.

Li GJ, Hyde KD, Zhao RL, Hongsanan S. et al. 2016a - Fungal diversity notes 253-366: taxonomic and phylogenetic contributions to fungal taxa. Fungal Diversity 78, 1-237.

Li W, Liu J, Bhat DJ, Camporesi E. et al. 2014 - Introducing the novel species, Dothiorella symphoricarposicola, from snowberry in Italy. Cryptogamie Mycology 35, 257-270.

Linaldeddu BT, Alves A, Phillips AJL. 2016b - Sardiniella urbana gen. et sp. nov., a new member of the Botryosphaeriaceae isolated from declining Celtis australis trees in Sardinian streetscapes. Mycosphere 7, 893-905.

Linaldeddu BT, Deidda A, Scanu B, Franceschini A. et al. 2015 - Diversity of Botryosphaeriaceae species associated with grapevine and other woody hosts in Italy, Algeria and Tunisia, with descriptions of Lasiodiplodia exigua and Lasiodiplodia mediterranea sp. nov. Fungal Diversity 71, 201-214.

Linaldeddu BT, Deidda A, Scanu B, Franceschini A. et al. 2016a - Phylogeny, morphology and pathogenicity of Botryosphaeriaceae, Diatrypaceae and Gnomoniaceae associated with branch diseases of hazelnut in Sardinia (Italy). European Journal of Plant Pathology 146, 259-279.

Linaldeddu BT, Maddau L, Franceschini A, Alves A, Phillips AJL. 2016c - Botryosphaeriaceae species associated with lentisk dieback in Italy and description of Diplodia insularis sp. nov. Mycosphere 7, 962-977.

Linaldeddu BT, Scanu B, Maddau L, Franceschini A. 2014 - Diplodia corticola and Phytophthora cinnamomi: the main pathogens involved in holm oak decline on Caprera island (Italy). Forest Pathology 44, 191-200.

Liu JK, Phookamsak R, Doilom M, Wikee S. et al. 2012 - Towards a natural classification of Botryosphaeriales. Fungal Diversity 57, 149-210.

Lopes A, Phillips AJL, Alves A. 2017 - Mating type genes in the genus Neofusicoccum: Mating strategies and usefulness in species delimitation. Fungal Biology 121, 394-404.

Marsberg A, Kemlera M, Jami F, Nagela JH. et al. 2017 - Botryosphaeria dothidea: A latent pathogen of global importance to woody plant health. Molecular Plant Pathology DOI: 10.1111/mpp.12495.

Mehl JWM, Slippers B, Roux J, Wingfield MJ. 2011 - Botryosphaeriaceae associated with Pterocarpus angolensis (kiaat) in South Africa. Mycologia 103, 534-553.

Mehl JWM, Slippers B, Roux J, Wingfield MJ. 2014 - Botryosphaeriaceae associated with dieback of Schizolobium parahyba trees in South Africa and Ecuador. Forest Pathology 44, 396408.

Nag Raj TR. 1993 - Coelomycetous Anamorphs with Appendage-bearing Conidia. Mycologue Publications, Waterloo.

Nylander JAA. 2004 - MrModeltest 2.0. Program distributed by the author. Evolutionary Biology Centre, Uppsala University.

Page RD. 1996 - TreeView: an application to display phylogenetic trees on personal computers. Computer Applications in the Biosciences 12, 357-358.

Pavlic-Zupanc D, Pis`kur B, Slippers B, Wingfield M, Jurc D. 2015 - Molecular and morphological characterization of Dothiorella species associated with dieback of Ostrya carpinifolia in Slovenia and Italy. Phytopathologia Mediterranea 54, 222-231.

Phillips AJL, Alves A, Abdollahzadeh J, Slippers B. et al. 2013 - The Botryosphaeriaceae: genera and species known from culture. Studies in Mycology 76, 51-167.

Phillips AJL, Alves A, Correia A, Luque J. 2005 - Two new species of Botryosphaeria with brown, 1-septate ascospores and Dothiorella anamorphs. Mycologia 97, 513-529.

Phillips AJL, Alves A, Pennycook SR, Johnston PR. et al. 2008 - Resolving the phylogenetic and taxonomic status of dark-spored teleomorph genera in the Botryosphaeriaceae. Persoonia $21,29-55$. 
Rayner RW. 1970 - A Mycological Colour Chart. Kew, UK: Commonwealth Mycological Institute \& British Mycological Society.

Rodríguez F, Oliver JL, Marín A, Medina JR. 1990 - The general stochastic model of nucleotide substitution. Journal of Theoretical Biology 142, 485-501.

Ronquist F, Huelsenbeck JP. 2003 - MrBayes 3: Bayesian phylogenetic inference under mixed models. Bioinformatics 19, 1572-1574.

Schoch CL, Shoemaker R, Seifert K, Hambleton S. et al 2006 - A multigene phylogeny of the Dothideomycetes using four nuclear loci. Mycologia 98, 1041-1052.

Slippers B, Boissin E, Phillips AJL, Groenewald JZ, Wingfield MJ, et al. 2013 - Phylogenetic lineages in the Botryosphaeriales: A systematic and evolutionary framework. Studies in Mycology 76, 31-49.

Slippers B, Roux J, Wingfield MJ, Van der Walt FJJ. et al. 2014 - Confronting the constraints of morphological taxonomy in the Botryosphaeriales. Persoonia 33, 155-168.

Slippers B, Wingfield MJ. 2007 - Botryosphaeriaceae as endophytes and latent pathogens of woody plants: diversity, ecology and impact. Fungal Biology Reviews 21, 90-106.

Spagnolo A, Marchi G, Peduto F, Phillips AJL, Surico G. 2011 - Detection of Botryosphaeriaceae within grapevine woody tissues by nested PCR, with particular emphasis on the Neofusicoccum parvum/N. ribis complex. European Journal of Plant Pathology 129, 485500.

Sutton BC, Marasas WFO. 1976 - Observations on Neottiosporina and Tiarosporella. Transactions of the British Mycological Society 67, 69-76.

Swofford DL. 2003 - PAUP*. Phylogenetic analysis using parsimony (*and other methods). Version 4.0. Sunderland, Massachusetts, Sinauer Associates.

Thambugala KM, Daranagama DA, Camporesi E, Singtripop C, et al. 2014 - Multi-locus phylogeny reveals the sexual state of Tiarosporella in Botryosphaeriaceae. Cryptogam Mycolologie 35, 359-367.

von Arx JA. 1987 - Plant-pathogenic fungi. J Cramer, Berlin.

White TJ, Bruns T, Lee S, Taylor J. 1990 - Amplification and direct sequencing of fungal ribosomal RNA genes for phylogenetics. In: Innis, M.A, Gelfand, D.H, Sninsky, J.J. \& White, T.J. (eds) PCR protocols: a guide to methods and applications. San Diego.

Yan JY, Xie Y, Zhang W, Wang, et al. 2013 - Species of Botryosphaeriaceae involved in grapevine dieback in China. Fungal Diversity 61, 221-236.

Yu L, Liu JN, Chen ZB, Xu S. 2016 - First report of stem die-back on Platanus $\times$ acerifolia caused by Neofusicoccum parvum in China. Plant Disease, (ja).

Zlatkovic M, Keca N, Wingfield MJ, Jami F, Slippers B. 2016 - Botryosphaeriaceae associated with the die-back of ornamental trees in the Western Balkans. Antonie Van Leeuwenhoek International of General Molecular Microbiology 109, 543-564. 